\title{
Towards High-Speed Energy-Efficient Pulse-Switching Networks Implemented in Carrier-Injection-Based Si-Photonics
}

\author{
Clemens J. Krückel ${ }^{1,2}$, Hanna Becker ${ }^{3}$, Yoojin Ban ${ }^{2}$, Martijn J.R. Heck ${ }^{3}$, \\ Joris Van Campenhout ${ }^{2}$, and Dries Van Thourhout ${ }^{1,2}$ \\ 1. Photonics Research Group, INTEC, Ghent University-IMEC, Ghent B-9000, Belgium \\ 2. IMEC, Kapeldreef 75, Leuven B-3001, Belgium \\ 3. Department of Engineering, Aarhus University, Denmark
}

We show that carrier injection based Si-photonics modulators [1] can form the basis for building compact, lowloss and power efficient reconfigurable networks, enabling the switching of ps-pulse trains with sub-GHz repetition rates. The use of pre-emphasis-based activation [2] permits ns-scale switching transitions. Although the steadystate energy consumption in this platform is well studied, the impact of the dynamic energy consumption for these ns switching periods is not well known. Here, we show pre-emphasis-based sub-ns transitions and present a novel large-signal analysis that allows the driving scheme optimization for energy-efficient pulse switching.
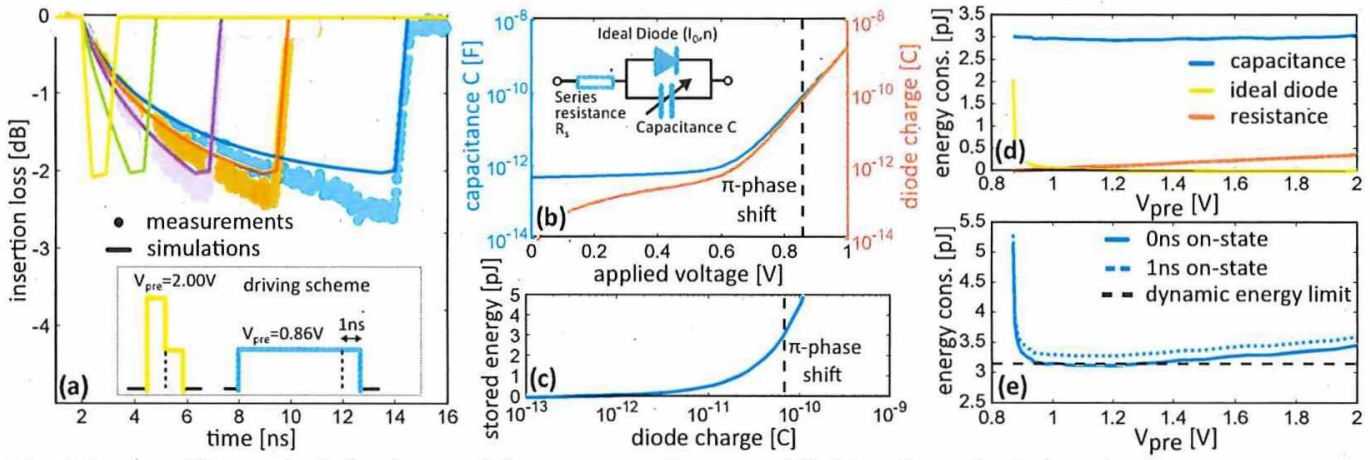

Figure 1: a) Impact of pre-emphasis level on modulator response time exemplified in a $1 \mathrm{~mm}$ pin-diode with a loss target comparable to a $\pi$-phase shift and $1 \mathrm{~ns}$ on-state. b) Measured capacitance and calculated stored diode charge versus applied voltage. c) Stored capacitive energy in relation to its stored diode charge. d) Contributions to dynamic energy consumption of large-signal model elements. e) Simulated energy consumption per switching operation versus pre-emphasis voltage. The dynamic energy limit equals the stored charge energy at $\pi$-phase shift.

The speed of pin-diode based switches depends on how fast charge carriers can be injected and removed from the diode. We studied these dynamics by monitoring the loss of $1 \mathrm{~mm}$ long pin diodes (Fig.1a ). We targeted a loss of $2 \mathrm{~dB}$, equivalent to a $\pi$-phase shift in these devices [3]. Five driving schemes are generated with constant 1 ns on-time and increasing pre-emphasis voltage level $\mathrm{V}_{\text {pre }}(0.86$ to $2.00 \mathrm{~V})$. As expected, a larger $\mathrm{V}_{\text {pre }}$ results in a faster response. We expect the same high-speed response in a switching network.

We compared these measurement results with simulations based on the large-signal model [4] shown in Fig.1b (inset). The resistance, diode and capacitance properties were extracted from DC-IV and $\mathrm{S}_{11}$ measurements. The measured voltage-dependent capacitance and the corresponding stored charge are visualized in Fig. $1 \mathrm{~b}$. The dashed line denotes the charge required for a $\pi$-phase shift in the modulator. Next, to calculate the energy that is stored in the capacitance for a given charge, we integrate charge over voltage $\left(E=\int_{0}^{V} C V d V\right)$. This stored energy corresponds to the energy required to charge the capacitance (dynamic energy dissipation). Fig. 1c indicates $\sim 3 \mathrm{pJ}$ is needed for a $\pi$-phase shift. This energy is independent on the activation scheme and representative for the modulator technology. In comparison, this value is $\sim 6 \mathrm{pJ}$ for carrier-depletion and $\sim 4 \mathrm{pJ}$ for carrier-accumulation technology [5], attesting the high modulation efficiency of the carrier-injection technology.

Fig.1d shows the additional contributions to the energy consumption, i.e. the charge leakage in the diode and

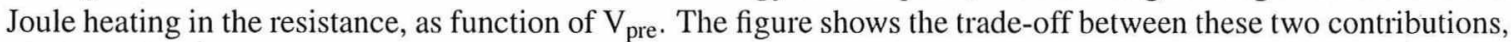
yielding a driving scheme with minimum energy consumption. This can be seen in Fig.1e where all contributions are combined. The solid curve, for no on-time, approaches the dynamic energy limit, which corresponds to the stored charge energy at $\pi$-phase shift. Thus, an optimized activation leads to energy savings of $\sim 40 \%$. Additionally, we see that the contribution from static-energy consumption is negligible for short activation times (1ns on-state). In conclusion, an optimized driving scheme leads to significant improvement of both speed and energy consumption in carrier-injection-based modulators which makes this platform suitable for energy-efficient pulse switching. This project has received funding from the European Unions Horizon 2020 research and innovation programme under grant agreement No 713481 .

References

[1] Dupuis al., "Nanosecond-Scale Mach-Zehnder-Based CMOS Photonics Switch Fabrics," J. Light. Technol. 35, 615-62 (2017)

[2] Xu et al., "12.5 Gbit/s carrier-injection-based silicon micro-ring silicon modulators,” Op. Exp. 15, 430-436 (2007)

[3] Soref et al., "Electrooptical Effects in Silicon," IEEE J. Quantum Electron. 23, 123-129 (1987)

[4] Pierret, "Semiconductor Device Fundamentals," Addison Wesley (1996)

[5] Azadeh et al., "Low $V_{\pi}$ Silicon photonics modulators with highly linear epitaxially grown ...," Op. Exp. 23, 23526-23550 (2015) 


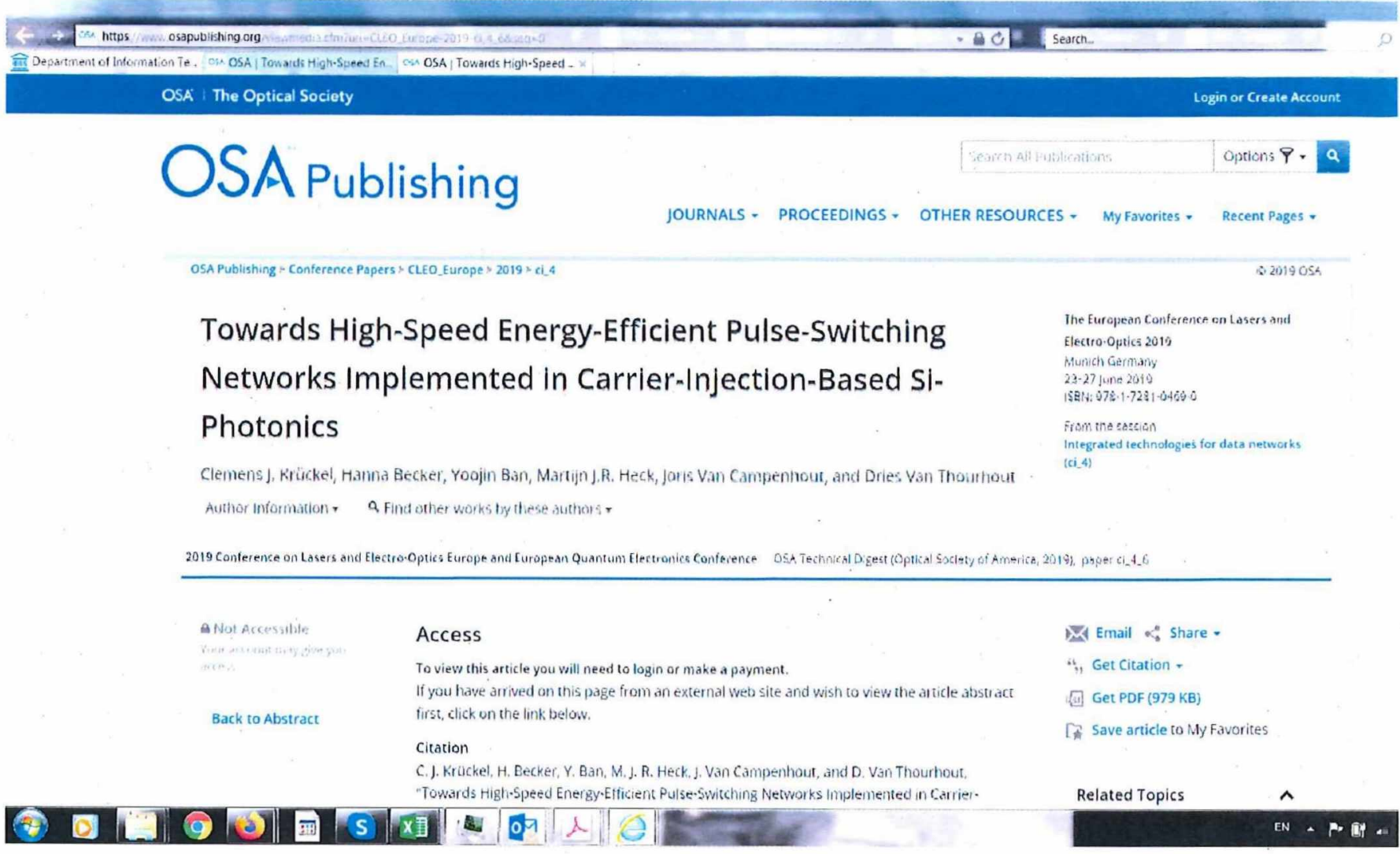

